\title{
Opportunities and Resistance to Signal-targeted Therapies in Pancreatic Cancer: \\ What Can We Learn from Proteomic Studies?
}

Cintas $\mathrm{C}^{1,2}$, Douche $\mathrm{T}^{1,2}$, Guillermet-Guibert $\mathrm{J}^{1,2 *}$.

Affiliation ${ }^{1}$ INSERM U1037, CRCT, Université Paul Sabatier, Toulouse; ${ }^{2}$ Laboratoire d'Excellence TouCAN.

* Corresponding author: Julie Guillermet-Guibert

CRCT UMR1037 INSERM-matUniversité Toulouse 3 - ERL5294 CNRS; 2 avenue Hubert Curien; Oncopole de Toulouse; CS 53717; 31037 TOULOUSE CEDEX 1 - FRANCE

Phone: +33-(0) 582741652

e-mail: julie.guillermet@inserm.fr

http://www.crct-inserm.fr/17-j-guillermet-guibert-sigdyn-group-pi3k-isoforms-signalling-

cancerogenesis-559.html

http://eupancreas.com/julie-guillermet-guibert

\section{Abstract}

In metastatic pancreatic cancer patients non eligible to surgery, signal-targeted therapies so far failed to show a significant amelioration of survival. These therapeutic options were tested in Phase II/III clinical trials mostly in combination with the reference treatment Gemcitabine. These innovative therapies aim at annihilating the oncogene dependency; they also aim at renormalizing the tumoral stroma to allow immune cell function or re-vascularisation. Transcriptomics and genomics large scale analysis show the great heterogeneity of pancreatic cancers and failed to clearly delineate specific oncogene dependency besides oncogenic Kras. In this review, we will describe the most recent proteomic data in pancreatic tumors and its metastasis, which could help at identifying their major signalling dependencies, as well as explain why they are intrinsically resistant to signal-targeted therapies. We will also discuss why PI3K signalling, as a paradigm of pro-tumorigenic cell signalling and of tumoral adaptative resistance to drugs, is a relevant target in this context.

\section{Key words}

Pancreatic cancer, proteomics, PI3K pathway, precision medicine, predictor of therapeutic response 


\section{Introduction}

So far, management of surgically resected pancreatic cancer provides the best chance of cure for the patients. This curative approach is only proposed in 15 to $20 \%$ of cases. Despite tumor resection, there is nevertheless a high rate of relapse. Local recurrence rates are greater than $50 \%$ after surgery. The 5 -year overall survival in resected patients is $28 \%$ and the median survival is 18 months. This high rate of recurrence is associated with the presence of micrometastasis at the time of surgery. This is why adjuvant chemotherapy is applied and significantly increases the survival of patients. A very recent meta-analysis of 14 articles generally shows that adjuvant treatment (chemotherapy or chemoradiotherapy) improves the survival of patients with pancreatic ductal adenocarcinoma (PDAC) [1]. One can therefore ask the question of the use of targeted therapies in this context, including those targeting the PI3K/AKT pathway [2]. Indeed, the lipid kinase PI3Ka was shown to drive pancreatic cancer initiation downstream the main driving oncogene of this cancer [3-6], oncogenic Kras, found mutated in more than $\mathbf{8 0} \%$ of all pancreatic cancer patients.

For the $80-85 \%$ of non-operable patients with locoregional or distant metastases (mainly liver and lung), chemotherapeutic treatment is applied to improve patients quality of life and survival by relieving symptoms of disease. Since 1997, gemcitabine monotherapy remains the standard palliative chemotherapy for patients with metastatic PDAC [7-10], given the many clinical failures to combine it with other agents [11]. Recently, the combination of gemcitabine and nab-paclitaxel (albumin-bound paclitaxel, the former allowing an improved pharmacokinetics, greater specificity of distribution in the tumor, higher intratumoral concentration, better efficacy) was superior to treatment with gemcitabine alone ( 8.5 months versus 6.7 months of survival), but with a higher toxicity $[12,13]$. The first major advance in the palliative treatment of pancreatic cancer dates from 2011 with FOLFIRINOX, a combination chemotherapy combining 5 -fluorouracil acid, irinotecan and oxaliplatin. Its use has become a standard in the treatment of metastatic PDAC for patients in good general condition. Its efficacy is superior to that of gemcitabine (mean overall survival of 11.1 months versus 6.7 months), but with more toxic side effects [14, 15]. Similarly, for these patients, how to select the most efficient targeted therapy is also an open question of the field.

The American Society of Clinical Oncology (ASCO) has published, in 2016, recommendations for the management of potentially curable PDACs, locally advanced PDACs, and metastatic PDACs (www.asco.org/guidelineswiki). Their recommendations for therapeutic interventions, including FOLFORINOX, irradiation and/or gemcitabine in combination with nab-paclitaxel, are based on relevant articles published between 2004 at 2015. However, it remains necessary and urgent to discover new targets and associated therapeutics, improve irradiation protocols and current therapeutic strategies by apprehending the mechanisms of intrinsic pancreatic resistance to antitumor drugs including targeted therapies. Here, we will discuss how the use of proteomic strategies will help to achieve these goals.

For this, key aspects will have to be answered by wider proteomics studies in the future. To name but a few:

I. Can we detect earlier PDAC with proteomics? Are the earlier detected tumors more sensitive to targeted therapies towards PDAC oncogenic dependency (e.g. PI3K)? 
II. Can we refine the current (epi)-genetic and genomic characterization of PDAC to better stratify patients with proteomics? This should also include a better understanding of its metastatic disease.

III. Can we identify new targets which take into account tumor-stroma heterotypic signalling with proteomics?

IV. Can we understand the specific resistance to targeted therapies of PDAC patients at the targetable protein level/modification (phosphorylation, ubiquitination) with proteomics?

\section{Targeted therapies in pancreatic cancer - what can we learn from the current clinical trials?}

Anticancer drugs fall into four broad categories based on their pharmacological action: conventional chemotherapy, radiopharmaceuticals, immunotherapies and inhibitors of (mostly) oncogenic mechanisms (but also angiogenesis) that include targeted therapies and hormone therapies (Figure 1A).

Figure1: Evolution of the use of targeted therapies in cancers for example in France. (A) Contribution in percentage of targeted therapies as compared to the therapeutic arsenal authorized in cancers excluding radiotherapies. Distribution (B) and number (C) of Marketing Authorization for Targeted Therapies. Adapted from INCa 2015 and completed with clinicaltrials.gouv. 
A

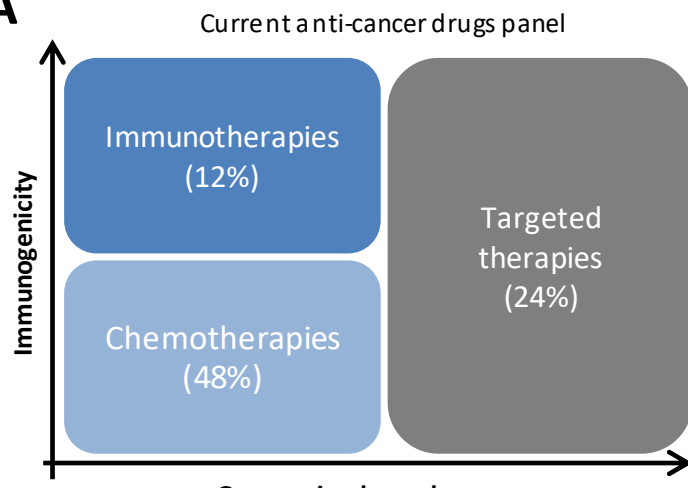

Oncogenic dependency

B Type of cancer

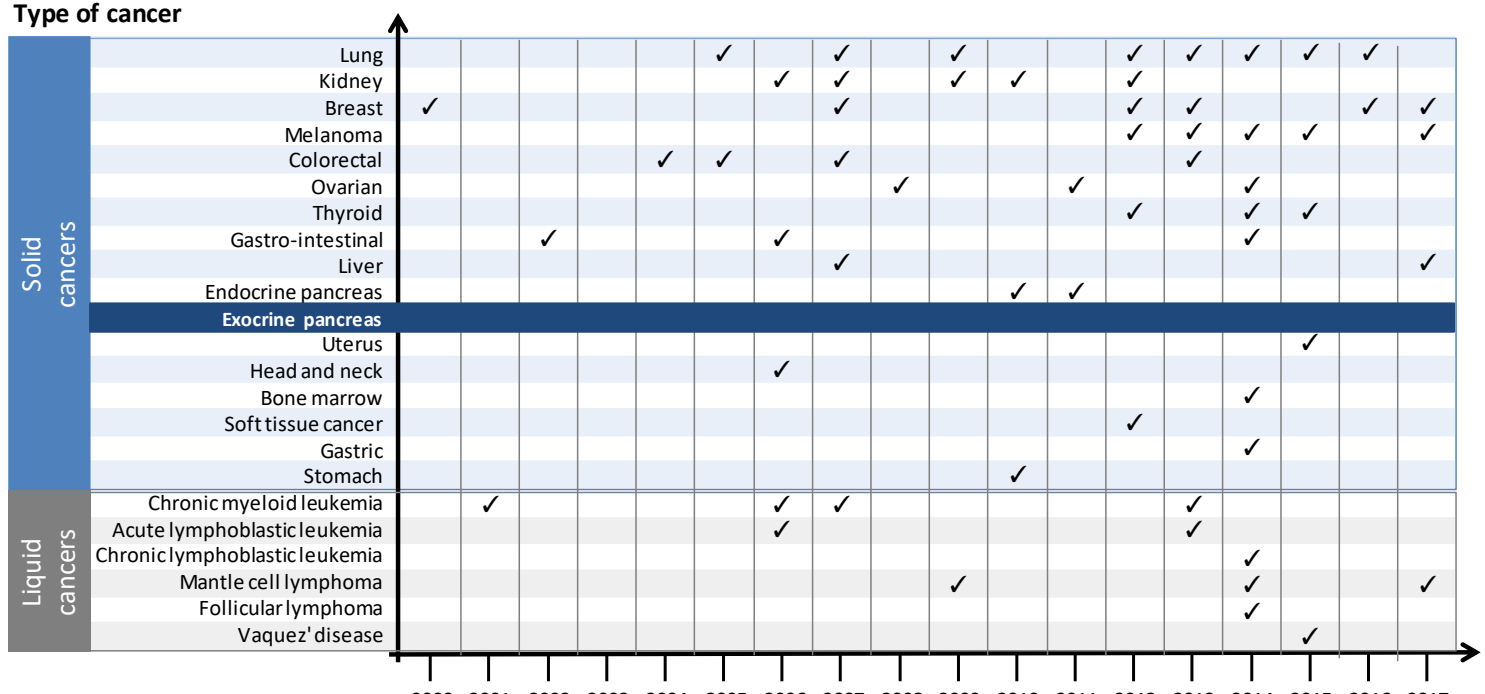

200020012002200320042005200620072008200920102011201220132014201520162017

Date of Marketing Authorizations with Targeted Therapies

C

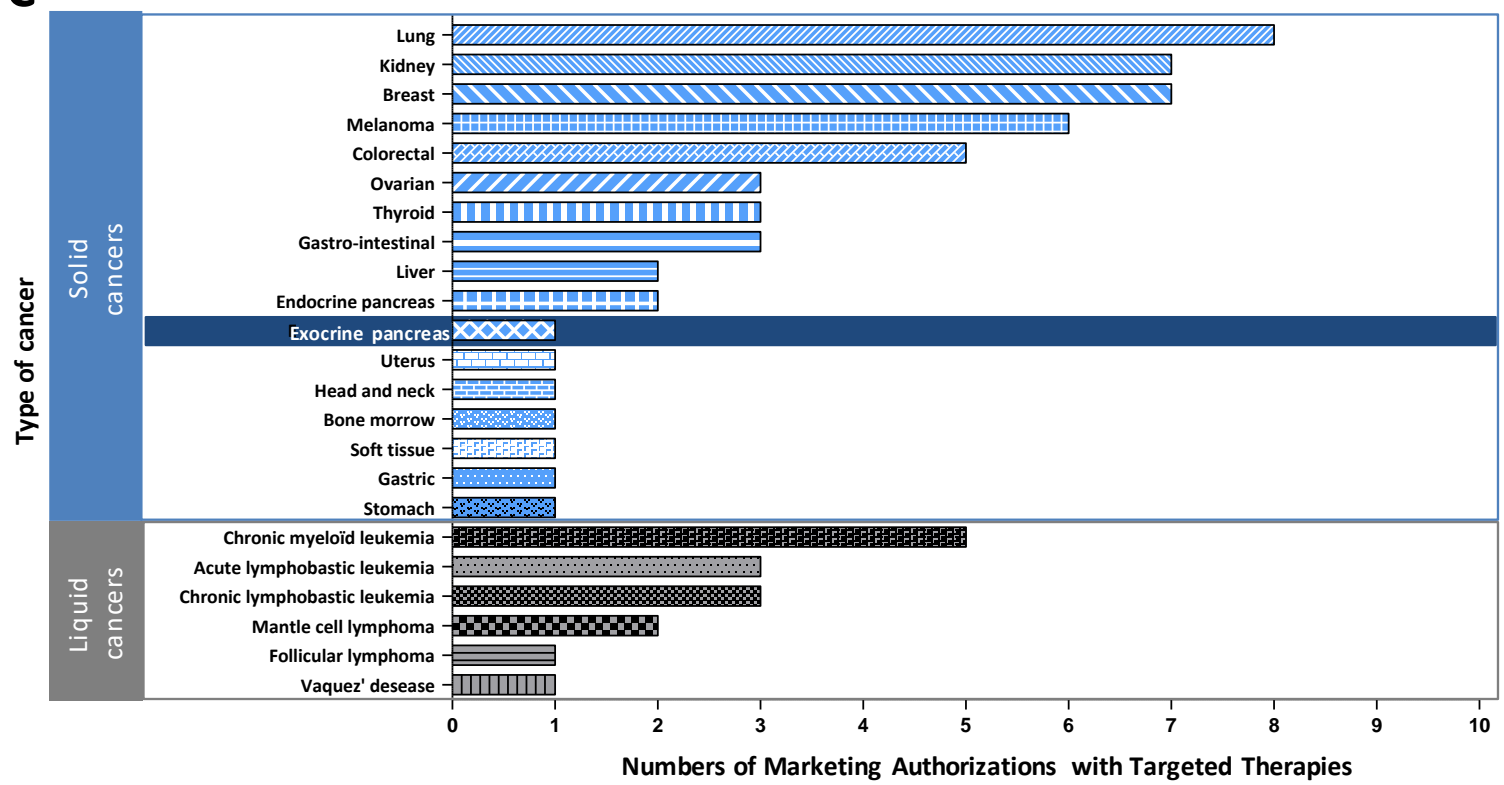


Targeted anti-cancer therapies are strategies that aim to block the growth and/or spread of tumor cells by specifically addressing some of their abnormalities. Their main mode of action goes through an inhibition of the mechanisms of oncogenesis with a higher specificity towards cancer cells or their microenvironment. These may be intracellular inhibitors (small chemical molecules such as protein or lipid kinase inhibitors) or extracellular inhibitors (biological drugs such as monoclonal antibodies to receptor tyrosine kinase RTK or their ligands) (Table 1).

Table 1: List of the 47 anti-cancer targeted therapies authorized for example in France. Adapted from INCa data and completed with Vidal.fr, updated in January 2018.

\begin{tabular}{|c|c|c|c|}
\hline \multicolumn{2}{|r|}{ Intracellular inhibitors } & \multicolumn{2}{|c|}{ Extracellular inhibitors } \\
\hline \multicolumn{2}{|c|}{ Inhibitors of protein(s) kinase(s) } & \multicolumn{2}{|c|}{ Ab diriged against RTK(s) } \\
\hline Name & Target(s) & Name & Target(s) \\
\hline Afatinib & EGFR & Cetuximab & Ab anti-EGFR \\
\hline Axitinib & VEGFR & Panitumumab & $\mathrm{Ab}$ anti-EGFR \\
\hline Osimertinib & EGFR & Pertuzumab & $\mathrm{Ab}$ anti-HER2 \\
\hline Bosutinib & Bcr-Abl, Src & Ramucirumab & $\mathrm{Ab}$ anti-VEGF \\
\hline Cabozantinib & $\begin{array}{c}\text { MET, AXL, VEGFR, GAS6, RET, ROS1, FLT3, } \\
\text { Tie2 }\end{array}$ & Trastuzumab & Ab anti-HER2 \\
\hline Ceritinib & ALK & Trastuzumab emtansine & $\mathrm{Ab}$ anti-HER2 \\
\hline Cobimetinib & MEK & \multicolumn{2}{|c|}{ Ab directed against ligand(s) } \\
\hline Crizotinib & ALK et MET & Aflibercept & $\mathrm{Ab}$ anti-VEGF \\
\hline Dabrafenib & RAF & BevAbizumab & Ab anti-VEGF \\
\hline Dasatinib & Bcr-Abl, Src & Denosumab & $\mathrm{Ab}$ anti-RANKL \\
\hline Erlotinib & EGFR & & \\
\hline Everolimus & mTOR & & \\
\hline Gefitinib & EGFR & & \\
\hline Ibrutinib & BTK & & \\
\hline Idelalisib & $\mathrm{p} 110 \delta$ (PI3K) & & \\
\hline Imatinib & Bcr-Abl, c-Kit, DDR1/2, CSF-1R, PDGFR & & \\
\hline Lapatinib & EGFR, ErbB2 & & \\
\hline Lenvatinib & VEGFR, FGFR, PDGFR & & \\
\hline Nilotinib & Bcr-Abl & & \\
\hline Nintedanib & PDGFR, FGFR, VEGFR, FLT3, Lck, Lyn, Src & & \\
\hline Olaparib & PARP & & \\
\hline Osimertinib & EGFR & & \\
\hline Palbociclib & CDK4/6 & & \\
\hline Pazopanib & VEGFR, c-Kit, PDGFR & & \\
\hline Ponatinib & Bcr-Abl & & \\
\hline Regorafenib & VEGFR, c-Kit, PDGFR & & \\
\hline Ribociclib & Cyclin D1/CDK4, CDK6 & & \\
\hline Ruxolitinib & JAK1/2 & & \\
\hline Sonidégib & SMO & & \\
\hline
\end{tabular}




\begin{tabular}{cc} 
Sorafenib & RAF, VEGFR, FGFR, c-Kit, PDGFR \\
\hline Sunitinib & VEGFR, c-Kit, c-Kit, CSF-1R, RET, PDGFR \\
\hline Temsirolimus & mTOR \\
\hline Tivozanib & VEGF \\
\hline Trametinib & MEK1/2 \\
\hline Vandetanib & VEGFR, EGFR, RET \\
\hline Venetoclax & Bcl2 \\
\hline Vemurafenib & ERK, BRAF \\
\hline Vismodégib & SMO
\end{tabular}

Targeted therapies are part of what is called "precision medicine". This term refers to a medicine that is based on a better knowledge of the biological mechanisms leading to the appearance and development of tumors. The use of these treatments is therefore guided, as far as possible, by the molecular characteristics of the tumor of each patient (for example: the state of differentiation of the tumor, genetic alterations such as mutations/overexpression of oncogenes, loss of function of tumor suppressor genes). The majority of targeted therapies are currently used as monotherapy (62\% in France). The first targeted therapy was approved there in 2000. This was trastuzumab, an antibody targeting the extracellular domain of the HER2 receptor in the treatment of HER2-positive metastatic breast cancer in monotherapy in patients already treated with at least two chemotherapies for their metastatic disease. End 2015, the French Cancer Health Institute INCa has identified 47 targeted therapies which have a Marketing Authorization (MA) in France for the treatment of cancer. One molecule only has an indication in PDAC (Figure 1B, 1C): inhibitors of the RTK EGFR. However, the efficiency of these molecules in PDAC remains modest, if compared to the spectacular action of targeted therapies in other aggressive solid tumors such as BRAF inhibitors in melanoma or EGFR inhibitors in lung cancers. Although overall survival (OS) and progression-free survival (PFS) improved slightly (case of EGFR inhibitor), combination with Gemcitabine individually provides no significantly different objective response (measurable response) as compared to placebo plus Gemcitabine [16, 17]. At present, in France, 38 clinical trials testing one or more anti-cancer molecules are in progress in pancreatic cancer (excluding neuroendocrine tumors), including novel 20 targeted therapies mostly used in combination with chemotherapy (Table 2). Targeted therapies against pancreatic microenvironment which are thought to contribute to pancreatic aggressiveness are not yet in phase I clinical trials.

Table 2: Ongoing clinical trials in pancreatic cancer in France. In gray: clinical trials associating a targeted therapy with a chemotherapy. In blue: clinical trials using a targeted therapy only. Adapted from clinical.gouv.fr, updated in August 2017.

\begin{tabular}{|c|c|c|c|c|c|}
\hline $\begin{array}{l}\text { Name of the } \\
\text { study }\end{array}$ & Molecule tested & Type of therapy & Type of drug & Phase & Pathologies \\
\hline $\begin{array}{l}\text { D081FC00001- } \\
\text { POLO }\end{array}$ & Olaparib vs placebo & Targeted therapy & Inhibitor of PARP & 3 & $\begin{array}{l}\text { PDAC metastatic with BRCA } \\
\text { mutation }\end{array}$ \\
\hline SIRINOX & Oxaliplatin + Irinotecan & Chemotherapy & $\begin{array}{l}\text { Platinum salts, DNA topoisomerase } \\
\text { I inhibitor }\end{array}$ & 1 & $\begin{array}{l}\text { Digestive adenocarcinoma } \\
\text { (pancreas, esophagus, stomach, } \\
\text { small intestine and biliary tract) }\end{array}$ \\
\hline PRODIGE 29 & FOLFIRINOX vs Gemcitabine & Chemotherapy & $\begin{array}{l}\text { Anti-metabolite, DNA } \\
\text { topoisomerase I inhibitor, Platinum } \\
\text { salts }\end{array}$ & 3 & PDAC locally advanced \\
\hline PAMELA-70 & FOLFIRINOX & Chemotherapy & $\begin{array}{l}\text { Anti-metabolite, DNA } \\
\text { topoisomerase I inhibitor, Platinum }\end{array}$ & 2 & PDAC metastatic \\
\hline
\end{tabular}




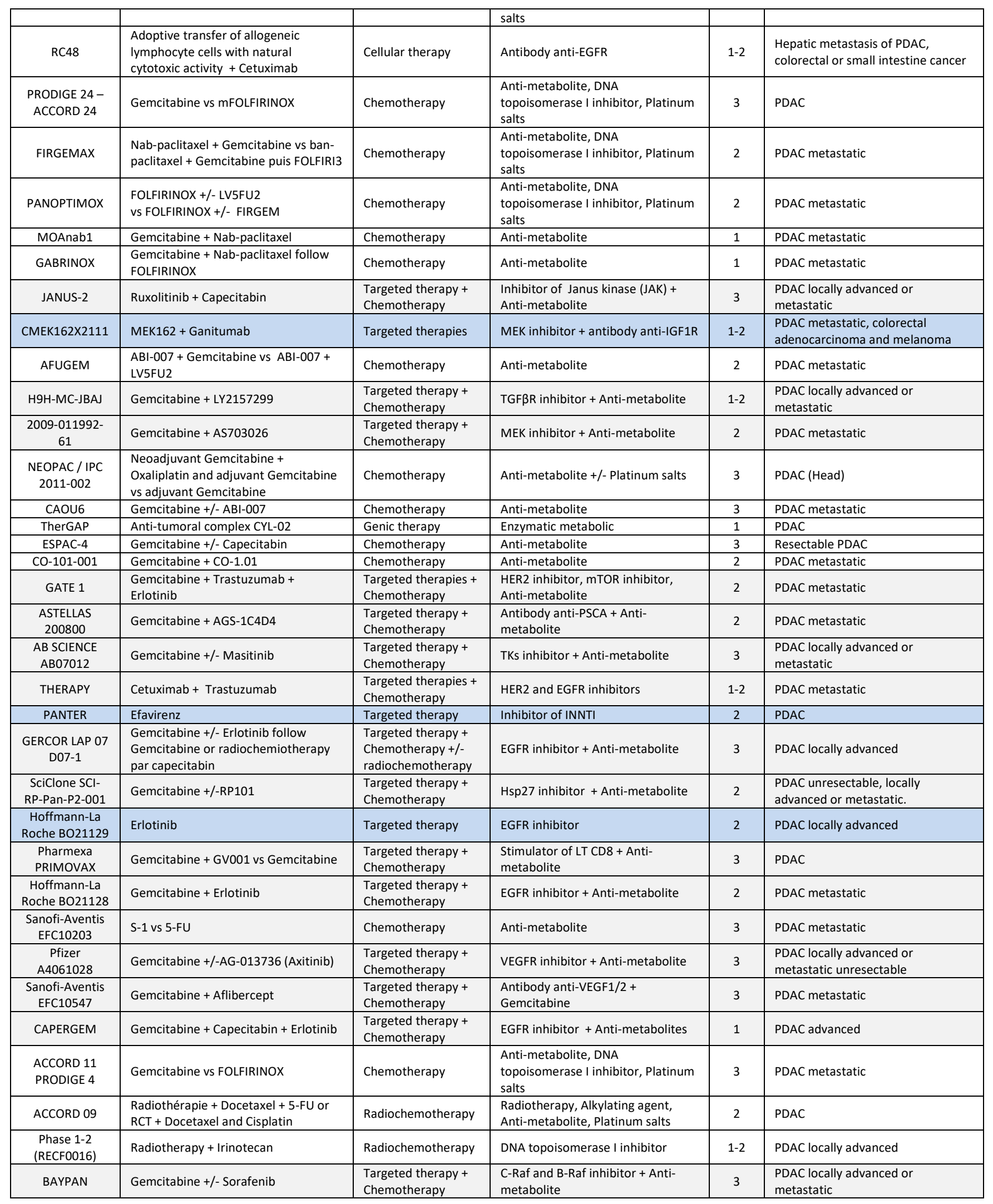

In PDAC, the efficacy of about 15 targeted therapies was evaluated in combination mostly with chemotherapy, Gemcitabine [18] and shown in details in Table 3. Despite an initial anti-tumor 
response of patients to these targeted therapies as described by an ameliorated general state of the patients, resistance is induced very quickly. Besides, the poor general state of patients before starting their treatment prevents the increase of doses to reach a better target coverage and is an issue when testing combination of several targeted therapies due to their increased toxicity. In the case of mTOR inhibitors, the clinical trials led to even worse survival, possibly due to an acceleration of resistance mechanisms as explained below. More generally, it was found that the improvement in patient survival is limited in time. More effective drug combinations which prevent resistance while sparing the general toxicity are needed.

Table 3: Outcome of the first targeted therapy clinical trials in pancreatic cancer. Some of the targeted therapies listed here did not have a high selectivity towards their targets [8-10].

\begin{tabular}{llcc} 
Target & Therapy & Patient number & $\begin{array}{c}\text { Mean survival (months) } \\
\text { (treatment versus chemo only) }\end{array}$ \\
\hline Telomerase & Gemcitabine + GV1001 & 1062 & 8.4 vs 6.9 \\
VEGF & Gemcitabine + Bevacizumab & 602 & 5.7 vs 6 \\
Kras & Gemcitabine + Tipifarnib & 688 & 6.3 vs 6 \\
EGFR & Gemcitabine + Cetuximab & 766 & 6.5 vs 6 \\
ErbB2 & Gemcitabine + Erlotinib & 569 & 6.24 vs 5.91 \\
Gastrin & Trastuzumab & 44 & 4.6 vs 5.4 \\
mTOR & Gastrazol + 5-FU & 98 & 3.6 vs 4.2 \\
PI3K/PLK & Gemcitabine + Everolimus & 29 & 4.5 vs 6.5 \\
Sonic Hedgehog & Gemcitabine + Rigosertib & 106 & 6.1 vs 6.4 \\
Notch3 & Gemcitabine + Vismodegib & 106 & 6.9 vs 6.1 \\
IGF1- R & Gemcitabine + Ganitumab & 122 & Not tolerated \\
MMP & Gemcitabine + Matrimastat & 800 & 7.0 vs 7.2 \\
JAK/STAT & Ruxolitinib + Capecitabin & 239 & 5.4 vs 5.4 \\
$\boldsymbol{\alpha}$-secretase & RO4929097 & 127 & 4.5 vs 4.2 \\
MEK1/ERK ${ }_{1 / 2}$ & Selumetinib versus Capecitabin & 18 & 4.1 \\
\hline
\end{tabular}

Indeed, although the majority of cells in a tumor are sensitive to a specific inhibitor, pancreatic cancer cells are mostly initially resistant (corresponding to a process called innate resistance). Also, adaptive resistance may appear in this clinical setting. One or more tumor subpopulations with different characteristics compared to the sensitive cells could emerge allowing them to survive and continue to proliferate in the presence of pharmacological inhibition, leading to a therapeutic failure $[19,20]$. The heterogeneity within tumor is thus a critical component of resistance mechanisms [21]. Technological progress and big data have led to a characterisation of pancreatic cancer's molecular identity (of mostly resected tumors) [22-25]. However, in contrast to other cancers such in lung cancer with EGFR mutations, these global approaches failed to identify simple therapeutic 
strategies based on a stratification of PDAC patients. Recent integrated omics approaches on all cancers points out that the search of genetic or genomic alterations is not sufficient to predict which patients will benefit from targeted therapies [26][69]. In contrast, proteomics appears better in predicting sensitivity to a targeted therapy (PI3K inhibitors) [26], suggesting that proteomic approaches could be a worthwhile strategy to investigate and to better understand the resistance to treatment of pancreatic cancer patients, so as to predict which therapy will be more efficient (Figure 2). Recent pioneering data which need to be complemented by wider studies argue for the effort to develop such challenging strategies in pancreatic cancer.

Figure 2: Proteomics for a better clinical care of pancreatic cancer patients.

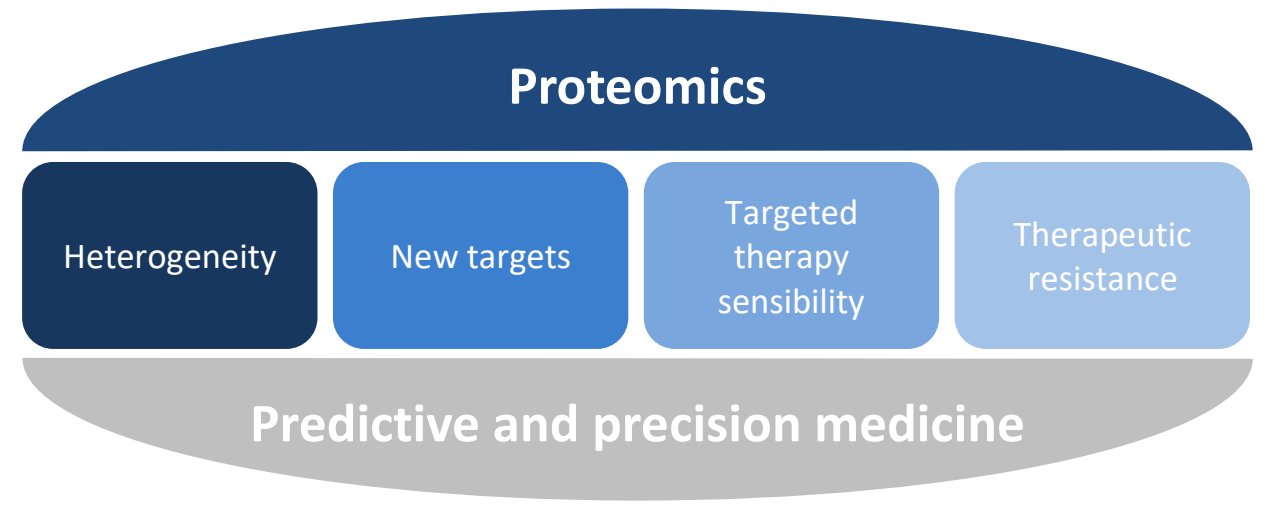

\section{Proteomics as a new way to improve clinical management of pancreatic cancer}

Proteomics consists of studying all the proteins of an organism, a biological fluid, an organ, a cell or even a cellular compartment. This set of proteins is called "proteome". The latter is a dynamic and complex entity. The proteome contains a much larger number of proteins than the genome contains genes. In human cells, an estimated 22.000 genes can yield up to one million proteins. Despite these approximations, it is considered that proteins represent about $60 \%$ of a cell. The study of proteins has grown dramatically during the 1990s, with the advent of mass spectrometers (Nobel Prize in Chemistry in 2002 to John Fenn and Koichi Tanaka) [27]. Mass spectrometry (MS) is an analytical method that aims to identify and separate molecules to be analyzed (small molecules, proteins, drugs...) with a very good resolution and sensitivity. It allows the qualitative and quantitative analysis of complex biological samples, which may contain thousands of proteins, some of which are present in small quantities. Post-translational modifications such as phosphorylation but also ubiquitination can be studied by these techniques. These post-translational alterations are key indicators of the activity of the proteins.

In pancreatic cancer, proteomics provides insight into proteome-related changes in the disease, such as observed protein changes in abundance, subcellular localization, post-translational modifications and cell signaling. The detection of these changes thus constitutes research interests ranging from the study of the mechanisms of initiation of the disease to the discovery of biomarkers. 
Published proteomic results for pancreatic cancer are derived from fluid samples (blood, plasma, serum, pancreatic juice, cystic fluid, in vitro cells conditions media) and/or solid samples (tumor tissue) from healthy versus sick patients (resected patients, representing a subpopulation of patients, see introduction), or patients at risk of developing pancreatic cancer (e.g. chronic pancreatitis CP) or murine models of PDAC. Tumor tissue is the main source of investigation of protein alterations associated with pancreatic cancer. These samples are mainly studied by mass spectrometry [28-32] Nevertheless, some articles report the use of a targeted proteomic/phosphoproteomic technique, called Reverse Phase Protein Array (RPPA) or "Reversed Phase Protein Chips" [33-35].

\section{Proteomic approaches to search new biomarkers of early diagnosis}

Early detection of pancreatic cancer offers hope for healing. Currently, tumor markers used clinically lack sensitivity and specificity. For example, the carbohydrate antigen marker CA-19-9 (secreted by exocrine cells and tumors) present in the serum makes it possible to estimate the pancreatic tumor progression during a treatment [36], but does not provide sufficient precision for the diagnosis of pancreatic cancer, because it is not very specific (high concentration in the sera of patients with acute and chronic pancreatitis, hepatitis and biliary obstruction).

Numerous studies report new specific biomarkers in the diagnosis of pancreatic cancer identified from pancreatic tissue lysate or liquid biopsies (such as secretome) [28, 29, 35, 37, 38, 39, 40-44]. Some biomarkers have been characterized and evaluated alone or in combination with CA19-9 on their ability to diagnose [45]. A major step forward has been reached very recently with the access to preneoplastic samples. So far, these samples were difficult to obtain due to the late diagnosis of this disease. Thrombospondin 2 detected in plasma as a biomarker alone or in combination with CA19-9 was validated ( $98 \%$ combination specificity, $87 \%$ sensitivity), to distinguish all the stages of the disease [45]. Studies on one type of the precursor lesions, intra-ductal papillary mucinous neoplasm (IPMN) and cystic precursor lesion of the pancreas, should lead to further insights towards the early diagnosis of pancreatic cancer $[46,47]$. Proteomic studies of biomarkers in patients at risk to develop pancreatic cancer, such as those with neo-onset diabetes, show that this condition impacts the diagnostic performance of CA19-9 [48]. These advances in early diagnosis are expected to increase the efficiency of the targeted therapies.

\section{Proteomics to grade the disease including the metastatic sites and identify new targets}

The majority of proteomic studies performed on pancreatic cancer to identify biomarkers does not discriminate the different grades of the tumors studied. However, 2D-DIGE technology performed on microdissections of human pancreatic tumors identified the calcium-binding protein S110A6 in moderately or poorly differentiated tumors [49]. In 2009, Sitek and its collaborators identified 86 differentially regulated proteins involved in pancreatic tumor progression only using microdissections [50]. These results are complemented by proteomic analysis in 2D-DIGE of microdissected murine PanIN cells (PanIN or Pancreatic Intraepithelial Neoplasia is a precursor lesion of PDAC) and plasma samples corresponding to different precancerous stages of genetically modified mice by Dufresne $M$ et al [51]. The identification of peptide signatures specific to each type of 
precancerous lesions would thus make it possible to discriminate them from normal pancreatic tissue. Similarly, other studies proposes classifications of pancreatic cancer cell lines based on the levels of tyrosine phosphorylation of RTKs, potentially identifying three groups of cell lines as low pTyr, enriched in RTK and mixed [52]. These data indicate that a combination of RTK is usually activated in pancreatic cancer, suggesting that single agent strategies towards RTKs are likely to be inefficient. If this classification could be performed in patient biopsies, this could allow predicting which targeted therapy could be efficient. To develop novel strategies targeting pancreatic cancer stem cells, large scale proteome analysis in cells or their secretome showed the importance of fatty acid synthesis and mevalonate pathways, as well as glycolysis, in these subtype of cells [53, 54]. These cells being possibly at the origin of tumor relapse under chemotherapy, discovering specific targets of this pool of cells could improve the current treatments. Recent data show that surface proteome of circulating exosomes provides unique opportunities to analyze the heterogeneity of the metastatic disease though the easy-to-access liquid biopsies [55]. Proteomics studies bring insight in metastatic PDAC biology, leading to the discovery of novel targets.

\section{Proteomic approaches to search new biomarkers of predictive response}

Proteomics is an adapted tool for predicting the response to targeted therapies by studying tumor heterogeneity and changes in signaling pathways during treatment [21]. Kim and his collaborators studied and apprehended the heterogeneity of three metastatic sites of pancreatic cancer (liver, lung and peritoneum) by creating three lines from each organ in the same patient [56]. This heterogeneity is characterized by changes in expression of the entire proteome and tyrosine kinase activity, in the three sites of metastasis. It is involved in differences in the sensitivity of neoplastic cells to targeted therapies. In contrast, in a large scale genomic and genetic analysis, genetic alterations in metastasis sites were found to be maintained as compared to the primary site of tumorigenesis [21]. Thus, this study highlights the interest of a personalized therapeutic combination targeting all the subclonal features of metastases, using proteomics to guide the therapeutic choice.

Also, proteomics is a powerful tool in the study of tumor cell/microenvironment cell signaling interactions and modifications. For example, Jorgensen's team has recently demonstrated from Krasmutated pancreatic cancer cells that this oncogenic tumor signaling activates a cell-autonomous signaling network but also non-autonomous activation of oncogenic signaling of stromal cells. Conversely, stromal cells can in turn modify and amplify oncogenic signaling in the same or other tumor cells. Thus, oncogenic signaling is no longer limited only to tumor cells but to the entire tumor compartment [57].

Hence, prediction of sensitivity to targeted therapies is influenced by, besides genetic and genomic alterations, the heterotypic tumor-stroma signalling; proteomic assessment in patientderived samples including all the cellular partners at stake appear to be the only way to assess it.

\section{Proteomic approaches to identify resistance mechanisms}

Global studies to understand the adaptative responses to targeted therapies in pancreatic cancer are starting to be published. Adaptative and reversible resistance to Kras inhibition in pancreatic 
cancer cells includes phosphorylation of focal adhesion pathway components, while strikingly no significant mutational or transcriptional changes were observed [58]. Temporal effects of paclitaxel on pancreatic cells via large scale proteomics highlight protein involved in mitochondrial function, survival (PI3K pathway) and cell cycle arrest as key resistance mechanism [59]. In PDAC, several negative feedback mechanisms of mTORC inhibitors have been identified using proteomics, explaining the disappointing results of the clinical trials on this target [38, 39, 57,59]. In other cancer settings, Hsu and colleagues have shown, for example, through a global phosphoproteome analysis approach (SILAC) that the mTORC1 complex is able to inhibit and degrade insulin and IGF-1 receptors through phosphorylation of the adapter protein Grb10 (Growth Factor Bound Protein 10) [60]. mTORC1 leads to the phosphorylation of Grb10 then to the ubiquitination and degradation of insulin and IGF receptors [61]. It is now generally agreed that to target PI3K/AKT/mTOR pathway, which is hyperactivated in $50 \%$ of all PDAC patients and associated with poor prognosis [4], hitting the upstream PI3K is a better strategy to prevent positive feedbacks due to mTOR inhibition. While some mechanisms of resistance to PI3K inhibitors were found [62, 63], it remains to be investigated in an integrated approach which mechanisms of resistance could still occur to allow a better efficiency of these innovative targeted therapies [2].

\section{Conclusion / Discussion / Perspectives}

To conclude, only more comprehensive knowledge on resistance mechanisms observed the level of the protein (target) induced by targeted therapies will allow the researchers and clinicians to develop effective therapeutic strategies adapted to each target / oncogenic pathway in each environment specific to each patient, abolishing, preventing or delaying the appearance of resistance.

Proteomics, and in particular phosphoproteomics (see Figure 2), are powerful and promising tools in:

i. the identification of early diagnostic and prognostic biomarkers;

ii. the identification of deregulated proteins and signaling pathways in the pathology;

iii. the identification of biomarkers predictive of the response to treatment;

iv. the identification of early resistance mechanism leading to the development of adapted combinatorial treatments.

It is difficult to perform in-depth and large-scale clinical studies on PDAC. However, recent advances in methods can advance PDAC proteomics both at the fundamental and clinical research stage. Besides the better understanding of oncogenic dependency of PDAC [64], current development of MS-based methods which need less material and are more quantitative [65], which are coupled to imaging $[66,67]$, or of non-MS based methods which are robust targeted proteomic approaches [68] together with the improvement of patient-derived ex vivo cultures better mimicking each patient situation will be instrumental to improve the management of pancreatic cancer patients.

Early diagnosis using proteomics is a growing field with already promising leads. The next challenge for PDAC proteomics will be to identify the appropriate biomarkers indicating which is the 
best targeted therapy strategy to use for each pancreatic cancer patient, and thus, to develop stratifications of patients according to each therapeutic approach.

\section{Abbreviations}

- ALK: Anaplastic Lymphoma Kinase;

- ASCO: American Society of Clinical Oncology;

- BTK: Bruton's tyrosine kinase;

- CA 19-9: Cancer Antigen 19-9;

- CDK4/6: Cyclin-Dependent Kinase;

- CSF-1R: Colony-stimulating factor 1 receptor;

- DDR1/2: Discoidin domain receptor tyrosine kinase 1/2;

- EGFR: Epidermal Growth Factor Receptor;

- FGFR: Fibroblast Growth Factor Receptor;

- FLT3: Fms-related tyrosine kinase 3;

- GAS6: Growth arrest-specific 6 ;

- Grb10: Growth Factor Bound Protein 10;

- Hsp27: Heat Shock Protein 27;

- IGF-1(R): Insulin Growth Factor (Receptor);

- IPMN: Intraductal papillary mucinous neoplasm;

- JAK: Janus Kinase;

- Lck: Lymphocyte-specific protein tyrosine kinase;

- MA: Marketing Authorization;

- MEK: Mitogen-Activated Protein Kinase;

- MET: Hepatocyte growth factor receptor;

- MS : Mass spectrometry;

- MTOR: mammalian target of Rapamycin;

- PanIN: Pancreatic Intraepithelial Neoplasia;

- PARP: Enzyme poly ADP ribose polymerase;

- PDAC: Pancreatic ductal adenocarcinoma;

- PDGFR: Platelet-derived growth factor receptor

- PI3K: Phosphoinositide 3-kinase;

- PSCA : Prostate stem cell antigen;

- OS: Overall survival;

- PFS: Progression-free survival;

- RANKL: Receptor activator of nuclear factor kappa-B ligand

- ROS1: c-ros oncogene 1;

- RPPA: Reverse Phase Protein Array;

- $\quad$ RTK: Receptor tyrosine kinase;

- Tie2: Tyrosine kinase with immunoglobulin and EGF homology domains;

- TGF $\beta R$ : Transforming growth factor $\beta$ receptor

- TK: Tyrosine Kinase;

- $\quad$ SILAC: Stable Isotope Labeling with Amino acids in Cell culture;

- SMO: Smoothened kinase;

- $\quad \operatorname{VEGF(R):~Vascular~endothelial~growth~factor~(receptor);~}$

- Vs: Versus 


\section{Glossary}

Heterotypic signalling

\section{Statement of conflict of interest}

The authors declare no conflict of interests.

\section{Author contributions}

CC and JGG wrote the article; CC, TD and JGG performed bibliographic search.

\section{Funding}

JGG's laboratory belongs to Toucan, Laboratoire d'Excellence, ANR, an integrated research program on Signal-targeted Drug Resistance. CC's salary was funded by French Charity Ligue Nationale Contre le Cancer (GB/MA/VSP-10443). JGG's laboratory for this topic was/is funded by Europe EU-ERG FP7 (270696 PaCa/PI3K), ARC (PJA20171206596), Toucan and MSCA-ITN/ETN PhD-PI3K (Project ID: 675392).

\section{Acknowledgments}

JGG is a member of COST action EU-Pancreas BM1204.

\section{Bibliography}

1. Xu, J., Bin Jiang, Ya Chen, Fu-Zhen Qi, Jian-Huai Zhang and Hang Yuan, Optimal adjuvant chemotherapy for resected pancreatic adenocarcinoma: a systematic review and network meta-analysis. Oncotarget, 2017.

2. Pons-Tostivint, E., B. Thibault, and J. Guillermet-Guibert, Targeting PI3K Signaling in Combination Cancer Therapy. Trends Cancer, 2017. 3(6): p. 454-469.

3. Baer, R., et al., Pancreatic cell plasticity and cancer initiation induced by oncogenic Kras is completely dependent on wild-type PI 3-kinase p110alpha. Genes Dev, 2014. 28(23): p. 262135.

4. Baer, R., et al., Implication of PI3K/Akt pathway in pancreatic cancer: When PI3K isoforms matter? Adv Biol Regul, 2015. 59: p. 19-35.

5. Eser, S., et al., Selective requirement of PI3K/PDK1 signaling for Kras oncogene-driven pancreatic cell plasticity and cancer. Cancer Cell, 2013. 23(3): p. 406-20.

6. Wu, C.Y., et al., PI3K regulation of RAC1 is required for KRAS-induced pancreatic tumorigenesis in mice. Gastroenterology, 2014. 147(6): p. 1405-16 e7.

7. Burris, H.A., 3rd, et al., Improvements in survival and clinical benefit with gemcitabine as firstline therapy for patients with advanced pancreas cancer: a randomized trial. J Clin Oncol, 1997. 15(6): p. 2403-13.

8. Adamska, A., A. Domenichini, and M. Falasca, Pancreatic Ductal Adenocarcinoma: Current and Evolving Therapies. Int J Mol Sci, 2017. 18(7). 
9. Danovi, S.A., H.H. Wong, and N.R. Lemoine, Targeted therapies for pancreatic cancer. Br Med Bull, 2008. 87: p. 97-130.

10. Barati Bagherabad, M., et al., Targeted Therapies in Pancreatic Cancer: Promises and Failures. J Cell Biochem, 2017.

11. Paulson, A.S., et al., Therapeutic advances in pancreatic cancer. Gastroenterology, 2013. 144(6): p. 1316-26.

12. Von Hoff, D.D., et al., Gemcitabine plus nab-paclitaxel is an active regimen in patients with advanced pancreatic cancer: a phase I/II trial. J Clin Oncol, 2011. 29(34): p. 4548-54.

13. De Vita, F., et al., NAB-paclitaxel and gemcitabine in metastatic pancreatic ductal adenocarcinoma (PDAC): from clinical trials to clinical practice. BMC Cancer, 2016. 16(1): p. 709.

14. Conroy, T., et al., FOLFIRINOX versus gemcitabine for metastatic pancreatic cancer. N EngI J Med, 2011. 364(19): p. 1817-25.

15. Gunturu, K.S., et al., FOLFIRINOX for locally advanced and metastatic pancreatic cancer: single institution retrospective review of efficacy and toxicity. Med Oncol, 2013. 30(1): p. 361.

16. Moore, M.J., et al., Erlotinib plus gemcitabine compared with gemcitabine alone in patients with advanced pancreatic cancer: a phase III trial of the National Cancer Institute of Canada Clinical Trials Group. J Clin Oncol, 2007. 25(15): p. 1960-6.

17. Rougier, P., et al., Randomised, placebo-controlled, double-blind, parallel-group phase III study evaluating aflibercept in patients receiving first-line treatment with gemcitabine for metastatic pancreatic cancer. Eur J Cancer, 2013. 49(12): p. 2633-42.

18. Di Marco, M., et al., State of the art biological therapies in pancreatic cancer. World J Gastrointest Oncol, 2016. 8(1): p. 55-66.

19. Burrell, R.A. and C. Swanton, Tumour heterogeneity and the evolution of polyclonal drug resistance. Mol Oncol, 2014. 8(6): p. 1095-111.

20. Almendro, V., A. Marusyk, and K. Polyak, Cellular heterogeneity and molecular evolution in cancer. Annu Rev Pathol, 2013. 8: p. 277-302.

21. Makohon-Moore, A.P., et al., Limited heterogeneity of known driver gene mutations among the metastases of individual patients with pancreatic cancer. Nat Genet, 2017. 49(3): p. 358366.

22. Waddell, N., et al., Whole genomes redefine the mutational landscape of pancreatic cancer. Nature, 2015. 518(7540): p. 495-501.

23. Bailey, P., et al., Genomic analyses identify molecular subtypes of pancreatic cancer. Nature, 2016. 531(7592): p. 47-52.

24. Sivakumar, S., et al., Master Regulators of Oncogenic KRAS Response in Pancreatic Cancer: An Integrative Network Biology Analysis. PLoS Med, 2017. 14(1): p. e1002223.

25. Collisson, E.A., et al., Subtypes of pancreatic ductal adenocarcinoma and their differing responses to therapy. Nat Med, 2011. 17(4): p. 500-3.

26. Zhang, Y., et al., A Pan-Cancer Proteogenomic Atlas of PI3K/AKT/mTOR Pathway Alterations. Cancer Cell, 2017. 31(6): p. 820-832 e3.

27. Fenn, J.B., et al., Electrospray ionization for mass spectrometry of large biomolecules. Science, 1989. 246(4926): p. 64-71.

28. Wehr, A.Y., et al., Relative quantification of serum proteins from pancreatic ductal adenocarcinoma patients by stable isotope dilution liquid chromatography-mass spectrometry. J Proteome Res, 2012. 11(3): p. 1749-58.

29. Gronborg, M., et al., Biomarker discovery from pancreatic cancer secretome using a differential proteomic approach. Mol Cell Proteomics, 2006. 5(1): p. 157-71.

30. Lee, Y.Y., et al., Phosphoproteomic analysis identifies activated MET-axis PI3K/AKT and MAPK/ERK in lapatinib-resistant cancer cell line. Exp Mol Med, 2013. 45: p. e64.

31. Wei, W., et al., Single-Cell Phosphoproteomics Resolves Adaptive Signaling Dynamics and Informs Targeted Combination Therapy in Glioblastoma. Cancer Cell, 2016. 29(4): p. 563-73. 
32. Chen, R., et al., Comparison of pancreas juice proteins from cancer versus pancreatitis using quantitative proteomic analysis. Pancreas, 2007. 34(1): p. 70-9.

33. Huang, Y.J., et al., Reverse-phase protein array analysis to identify biomarker proteins in human pancreatic cancer. Dig Dis Sci, 2014. 59(5): p. 968-75.

34. Grote, T., et al., Validation of reverse phase protein array for practical screening of potential biomarkers in serum and plasma: accurate detection of CA19-9 levels in pancreatic cancer. Proteomics, 2008. 8(15): p. 3051-60.

35. Mustafa, S., et al., Comparison of the tumor cell secretome and patient sera for an accurate serum-based diagnosis of pancreatic ductal adenocarcinoma. Oncotarget, 2017. 8(7): p. 11963-11976.

36. Wakabayashi, T., et al., Diagnostic significance of cancer-associated carbohydrate antigen (CA19-9) concentrations in pancreatic juice: analysis in pure pancreatic juice collected by endoscopic aspiration and immunohistochemical study in chronic pancreatitis. Pancreas, 1993. 8(2): p. 151-9.

37. Fakelman, F., et al., New pre-analytical approach for the deep proteome analysis of sera from pancreatitis and pancreas cancer patients. Arch Physiol Biochem, 2010. 116(4-5): p. 208-17.

38. Sun, C., et al., Proteome-based biomarkers in pancreatic cancer. World J Gastroenterol, 2011. 17(44): p. 4845-52.

39. Takano, S., et al., Increased circulating cell signalling phosphoproteins in sera are useful for the detection of pancreatic cancer. Br J Cancer, 2010. 103(2): p. 223-31.

40. Lin, C., et al., ITRAQ-based quantitative proteomics reveals apolipoprotein A-I and transferrin as potential serum markers in CA19-9 negative pancreatic ductal adenocarcinoma. Medicine (Baltimore), 2016. 95(31): p. e4527.

41. Park, J., et al., Large-scale clinical validation of biomarkers for pancreatic cancer using a mass spectrometry-based proteomics approach. Oncotarget, 2017.

42. Saraswat, M., et al., Comparative proteomic profiling of the serum differentiates pancreatic cancer from chronic pancreatitis. Cancer Med, 2017.

43. Britton, D., et al., Quantification of pancreatic cancer proteome and phosphorylome: indicates molecular events likely contributing to cancer and activity of drug targets. PLoS One, 2014. 9(3): p. e90948.

44. Crnogorac-Jurcevic, T., et al., Proteomic analysis of chronic pancreatitis and pancreatic adenocarcinoma. Gastroenterology, 2005. 129(5): p. 1454-63.

45. Jenkinson, C., et al., Evaluation in pre-diagnosis samples discounts ICAM-1 and TIMP-1 as biomarkers for earlier diagnosis of pancreatic cancer. J Proteomics, 2015. 113: p. 400-2.

46. Ilies, M., et al., Plasma protein profiling of patients with intraductal papillary mucinous neoplasm of the pancreas as potential precursor lesions of pancreatic cancer. Clin Chim Acta, 2018. 477: p. 127-134.

47. Jabbar, K.S., et al., Highly Accurate Identification of Cystic Precursor Lesions of Pancreatic Cancer Through Targeted Mass Spectrometry: A Phase Ilc Diagnostic Study. J Clin Oncol, 2018. 36(4): p. 367-375.

48. Jenkinson, C., et al., Decreased Serum Thrombospondin-1 Levels in Pancreatic Cancer Patients Up to 24 Months Prior to Clinical Diagnosis: Association with Diabetes Mellitus. Clin Cancer Res, 2016. 22(7): p. 1734-1743.

49. Shekouh, A.R., et al., Application of laser capture microdissection combined with twodimensional electrophoresis for the discovery of differentially regulated proteins in pancreatic ductal adenocarcinoma. Proteomics, 2003. 3(10): p. 1988-2001.

50. Sitek, B., et al., Analysis of the pancreatic tumor progression by a quantitative proteomic approach and immunhistochemical validation. J Proteome Res, 2009. 8(4): p. 1647-56.

51. Ligat, L., et al., Pancreatic preneoplastic lesions plasma signatures and biomarkers based on proteome profiling of mouse models. Br J Cancer, 2015. 113(11): p. 1590-8.

52. Humphrey, E.S., et al., Resolution of Novel Pancreatic Ductal Adenocarcinoma Subtypes by Global Phosphotyrosine Profiling. Mol Cell Proteomics, 2016. 15(8): p. 2671-85. 
53. Brandi, J., et al., Secretome protein signature of human pancreatic cancer stem-like cells. J Proteomics, 2016. 136: p. 1-12.

54. Brandi, J., et al., Proteomic analysis of pancreatic cancer stem cells: Functional role of fatty acid synthesis and mevalonate pathways. J Proteomics, 2017. 150: p. 310-322.

55. Castillo, J., et al., Surfaceome profiling enables isolation of cancer-specific exosomal cargo in liquid biopsies from pancreatic cancer patients. Ann Oncol, 2018. 29(1): p. 223-229.

56. Kim, M.S., et al., Heterogeneity of pancreatic cancer metastases in a single patient revealed by quantitative proteomics. Mol Cell Proteomics, 2014. 13(11): p. 2803-11.

57. Tape, C.J., et al., Oncogenic KRAS Regulates Tumor Cell Signaling via Stromal Reciprocation. Cell, 2016. 165(7): p. 1818.

58. Chen, P.Y., et al., Adaptive and reversible resistance to Kras inhibition in pancreatic cancer cells. Cancer Res, 2017.

59. Wang, X., et al., Temporal Effects of Combined Birinapant and Paclitaxel on Pancreatic Cancer Cells Investigated via Large-scale, Ion-Current-Based Quantitative Proteomics (IonStar). Mol Cell Proteomics, 2018.

60. Hsu, P.P., et al., The mTOR-regulated phosphoproteome reveals a mechanism of mTORC1mediated inhibition of growth factor signaling. Science, 2011. 332(6035): p. 1317-22.

61. Yu, Y., et al., Phosphoproteomic analysis identifies Grb10 as an mTORC1 substrate that negatively regulates insulin signaling. Science, 2011. 332(6035): p. 1322-6.

62. Alagesan, B., et al., Combined MEK and PI3K inhibition in a mouse model of pancreatic cancer. Clin Cancer Res, 2015. 21(2): p. 396-404.

63. Junttila, M.R., et al., Modeling targeted inhibition of MEK and PI3 kinase in human pancreatic cancer. Mol Cancer Ther, 2015. 14(1): p. 40-7.

64. Ying, H., et al., Genetics and biology of pancreatic ductal adenocarcinoma. Genes Dev, 2016. 30(4): p. 355-85.

65. Dalla Pozza, E., et al., Trichostatin A alters cytoskeleton and energy metabolism of pancreatic adenocarcinoma cells: An in depth proteomic study. J Cell Biochem, 2017.

66. Gruner, B.M., et al., MALDI imaging mass spectrometry for in situ proteomic analysis of preneoplastic lesions in pancreatic cancer. PLoS One, 2012. 7(6): p. e39424.

67. Gruner, B.M., et al., Modeling Therapy Response and Spatial Tissue Distribution of Erlotinib in Pancreatic Cancer. Mol Cancer Ther, 2016. 15(5): p. 1145-52.

68. Hilhorst, R., et al., Peptide microarrays for detailed, high-throughput substrate identification, kinetic characterization, and inhibition studies on protein kinase A. Anal Biochem, 2009. 387(2): p. 150-61. 